ANNALES

POLONICI MATHEMATICI

LI (1990)

\title{
On improper isolated intersection in complex analytic geometry
}

by R. Achilles (Halle), P. Tworzewski, T. Winiarski (Kraków)

\section{Zdzislaw Opial in memoriam}

\begin{abstract}
In the paper, we present an analytic approach to the intersection multiplicity in the isolated improper complex analytic case and we compare it with Samuel's multiplicity.
\end{abstract}

1. Introduction. There are several approaches to the intersection but most of them are constructed for algebraic schemes and it is not clear which of them can be extended to intersection of locally analytic subsets of a complex manifold (cf. [5], Example 19.25, p. 383).

In paper [4], the intersection theory for analytic subsets of complex manifolds was presented. The multiplicity for proper isolated intersection is defined by "moving to general position" and then counting points of intersection. Since in the proper case there exists a good system of axioms of intersection multiplicity (see e.g. [14], [19]), all theories yield the same multiplicity.

Our aim is to relate to Samuel's [12] and Weil's [19] idea of the diagonal construction to define intersection multiplicity of two, or more than two, purely dimensional locally analytic subsets $X, Y$ of a complex manifold $M$ at an isolated point of $X \cap Y$.

We define

$$
i(X \cdot Y ; a)=\tilde{i}((X \times Y) \cdot \Delta,(a, a)),
$$

where $\Delta$ is the diagonal in $M \times M$,

$$
\tilde{i}((X \times Y) \cdot \Delta ;(a, a))=\inf \{i((X \times Y) \cdot V ;(a, a))\}
$$

and infimum is taken over all locally analytic subsets $V$ of pure dimension $2 \operatorname{dim} M-\operatorname{dim} X-\operatorname{dim} Y$ such that the germ $\Delta_{(a, a)}$ of $\Delta$ at $(a, a)$ is contained in the germ $V_{(a, a)}$ and $(a, a)$ is an isolated point of $(X \times Y) \cap V$.

Since the intersection of $X \times Y$ and $V$ is proper at $(a, a), i((X \times Y) \cdot V ;(a, a))$ is considered here as the intersection multiplicity in the sense of Draper [4]. 
Our presentation is novel in three respects. First, it is for the local analytic case. Second, in earlier constructions (see e.g. [2]) extensions $V$ of $\Delta$ are considered to be linear only. Third, we present here a full characterization of $V$ for which

$$
i((X \times Y) \cdot V ;(a, a))=\tilde{i}((X \times Y) \cdot \Delta,(a, a)) .
$$

Thus, instead of the word "generic" or "generic enough" we describe, in analytic context, its meaning.

As a simple consequence of the approach presented, we obtain (see Theorem 5.6) that also in the improper case we have

$$
i(X \cdot Y ; a) \geqslant \operatorname{deg}_{a} X \cdot \operatorname{deg}_{a} Y
$$

with equality if and only if $C_{a}(X) \cap C_{a}(Y)=\{0\}$ (for the proper intersection, see e.g. [5], [22]).

We end this paper by proving that the multiplicity of the primary ideal of the diagonal $\Delta$ in the local ring $O_{X \times Y,(a, a)}$ and $i(X \cdot Y ; a)$ are equal. This together with van Gastel's results (see [6], [8]) give possibility to compare our approach with other ones.

2. Relative tangent cone. Let $A, B$ be analytic subsets of an open neighbourhood $U$ of a point $a \in C^{m}$ such that $a$ is an isolated point of $A \cap B$.

The relative tangent cone $C_{a}(A, B)$ of the sets $A, B$ at $a$ is defined to be the set of $v \in C^{m}$ with the property: There exist sequences $\left\{x_{v}\right\}$ of points of $A,\left\{y_{v}\right\}$ of points of $B$ and $\left\{\lambda_{v}\right\}$ of complex numbers such that $x_{v} \rightarrow a, y_{v} \rightarrow a$ and $\lambda_{v}\left(y_{v}-x_{v}\right) \rightarrow v$ as $v \rightarrow \infty$.

If $B=\{a\}$ then $C_{a}(A, B)=C_{a}(A)$ is the tangent cone of $A$ at $a$ (in the sense of Whitney, [20], p. 510). The cone $C_{a}(A, B)$ is clearly a closed cone from 0 . Since $C_{a}(A, B)$ depends only on germs of $A$ and $B$ at $a$, we shall assume that $U$ is distinguished with respect to $A, B$, i.e., $\bar{U}$ is compact, $A \cap B=\{a\}$ and $(\partial A \cap \bar{B}) \cup(\bar{A} \cap \partial B)=\varnothing$, where $\partial A=\bar{A} \backslash A$ and the closure of $A$ is taken in $C^{m}$.

LEMMA 2.1. If $A, B$ are analytic in a neighbourhood $U$ of a, distinguished with respect to $A, B$ at $a$, then

$$
B-A=\left\{y-x \in C^{m} \mid y \in B, x \in A\right\}
$$

is analytic in an open neighbourhood of 0 in $C^{m}$,

$$
\operatorname{dim}(B-A)_{0}=\operatorname{dim}(A)_{a}+\operatorname{dim}(B)_{a} \quad \text { and } \quad C_{a}(A, B)=C_{0}(B-A),
$$

where $\operatorname{dim}(A)_{a}$ denotes the dimension of the germ $(A)_{a}$ of the set $A$ at a. If $A, B$ are of pure dimension, so is $B-A$ near 0 .

Proof. Without loss of generality we may assume that $a=0$. The mapping

$$
f: \boldsymbol{C}^{m} \times \boldsymbol{C}^{\boldsymbol{m}} \ni(x, y) \rightarrow(x, y-x) \in \boldsymbol{C}^{\boldsymbol{m}} \times \boldsymbol{C}^{m}
$$


is a linear automorphism of $\boldsymbol{C}^{m} \times \boldsymbol{C}^{m}$. Since 0 is an isolated point of $\left(C^{m} \times\{0\}\right) \cap f(A \times B)$, there exist open neighbourhoods $\Omega_{1}, \Omega_{2}$ of 0 in $C^{m}$ such that

(i) $\bar{\Omega}_{1}, \bar{\Omega}_{2}$ are compact subsets of $C^{m}$,

(ii) $\left(\bar{\Omega}_{1} \times\{0\}\right) \cap f(A \times B)=\{0\}$,

(iii) $\left(\partial \Omega_{1} \times \bar{\Omega}_{2}\right) \cap f(A \times B)=\varnothing$ and

(iv) $f(A \times B)$ is analytic in an open subset of $C^{m} \times C^{m}$ containing $\bar{\Omega}_{1} \times \bar{\Omega}_{2}$.

Let $W=f(A \times B) \cap\left(\Omega_{1} \times \Omega_{2}\right)$. It follows from (iii) that the restriction $\pi \mid W$ of the natural projection

$$
\pi: C^{m} \times C^{m} \ni(x, y) \rightarrow y \in C^{m}
$$

is proper. Since $\pi(W)=B-A$ near 0 , by Remmert's theorem, $B-A$ is analytic in an open neighbourhood of 0 and its germ at 0 has dimension $\operatorname{dim}(A)_{0}+\operatorname{dim}(B)_{0}$.

The inclusion $C_{a}(A, B) \subset C_{0}(B-A)$ is trivial.

To prove that $C_{0}(B-A) \subset C_{a}(A, B)$, it is enough to observe that if $0 \neq z_{v}=y_{v}-x_{v} \rightarrow 0$ with $x_{v} \in A, y_{v} \in B, v=1,2, \ldots$, then, by assumption that $U$ is distinguished with respect to $A, B$ at $A, x_{v} \rightarrow a$ and $y_{v} \rightarrow a$.

Let $v \in C_{a}(A, B), x_{v}, y_{v}, \lambda_{v}, v=1,2, \ldots$, be the same as in the definition of $C_{a}(A, B)$ and let $f: U \rightarrow C^{k}$ be a holomorphic mapping. By standard calculation we have

$$
\lambda_{v}\left(f\left(y_{v}\right)-f\left(x_{v}\right)\right) \rightarrow f^{\prime}(a) \cdot v
$$

Thus, if $a$ is an isolated point of $A \cap B$ and $f: A \cup B \rightarrow C^{k}$ is holomorphic, we may define $f^{\prime}(a) \cdot v$ for $v \in C_{a}(A, B)$ by means of extension of $f$ through a neighbourhood of $a$. By (2.3) the result is independent of the holomorphic extension chosen.

LeMMA 2.2 (cf. [20], Lemma 9.1). Let $A, B$ be analytic subsets of an open neighbourhood of $a \in C^{m}$ with $A \cap B=\{a\}$ and let $C, D$ be analytic subsets of an open subset of $C^{k}$ with $C \cap D=\{b\}$. If $f: A \cup B \rightarrow C \cup D$ is holomorphic and $f(A) \subset C, f(B) \subset D$, then

$$
f^{\prime}(a)\left(C_{a}(A, B)\right) \subset C_{b}(C, D) .
$$

Proof. Follows from (2.3).

LeMma 2.3 (cf. [20], Lemma 9.3). Suppose A, B satisfies the assumptions of Lemma 2.2. If $f: A \cup B \rightarrow C^{k}$ is holomorphic and, for some $\eta>0$,

$$
\|f(y)-f(x)\| \geqslant \eta\|y-x\|, \quad x \in A, y \in B,
$$

then $C=f(A)$ and $D=f(B)$ are analytic in an open neighbourhood of $b=f(a)$ and

$$
f^{\prime}(a)\left(C_{a}(A, B)\right)=C_{b}(C, D) .
$$

Proof. It follows from [20], Lemma 9.3, that $C$ and $D$ are analytic near $b$. 
To complete the proof it is enough to show, because of Lemma 2.1, that for every $w \in C_{b}(C, D)$ there exists $v \in C_{a}(A, B)$ such that $f^{\prime}(a) \cdot v=w$.

If $w=0$, set $v=0$. Now suppose $w \neq 0$ and suppose that $C, D$ are analytic in a neighbourhood of $b$ distinguished with respect to $C, D$ at $b$, and the same for $A, B$. Since $w \in C_{b}(C, D)$, there exist sequences: $0 \neq \lambda_{v} \in C, x_{v} \in A, y_{v} \in B$, $v=1,2, \ldots$, such that $f\left(y_{v}\right)-f\left(x_{v}\right) \neq 0, f\left(x_{v}\right) \rightarrow b, f\left(y_{v}\right) \rightarrow b$ and $\lambda_{v}\left(f\left(y_{v}\right)\right.$ $\left.-f\left(x_{v}\right)\right) \rightarrow w$. By (2.5), $0 \neq y_{v}-x_{v} \rightarrow 0$. Hence, since $A, B$ are analytic in a distinguished neighbourhood of $a$, we have $y_{v} \rightarrow a$ and $x_{v} \rightarrow a$. Since, by (2.5), the sequence $\left\{\lambda_{v}\left(y_{v}-x_{v}\right)\right\}$ is bounded, using a subsequence if necessary, we may suppose that $\lambda_{v}\left(y_{v}-x_{v}\right) \rightarrow v$. Now, by $(2.3), f^{\prime}(a) \cdot v=w$, as required.

Lemma 2.4. Suppose that $a \in A, L_{1}$ and $L_{2}$ are linear subspaces of $C^{m}$ such that $L_{1} \cap L_{2}=\{0\}, C^{m}=L_{1}+L_{2}$ and $\left(L_{2}+a\right) \cap A=\{a\}$. If

$$
\pi: L_{1}+L_{2} \ni x_{1}+x_{2} \rightarrow x_{1} \in L_{1}
$$

is the natural projection onto $L_{1}$, then for some neighbourhood $U$ of $a$ in $C^{m}, w=\pi(A \cap U)$ is analytic near $a_{1}=\pi(a)$ in $L_{1}$ and

$$
C_{a}\left(A, L_{2}+a\right)=C_{a_{1}}(W)+L_{2} .
$$

Proof. If $a$ is an isolated point of $A$ then (2.7) is trivial. Now suppose that $\operatorname{dim}(A)_{a}>0$ and $a=0$.

By Lemma $2.2, \pi\left(C_{a}\left(A, L_{2}+a\right)\right) \subset C_{a_{1}}(W)$. Hence

$$
C_{a}\left(A, L_{2}+a\right) \subset C_{a_{1}}(W)+L_{2} \text {. }
$$

Suppose $v=v^{\prime}+v^{\prime \prime}$ with $v^{\prime} \in C_{a_{1}}(W), v^{\prime \prime} \in L_{2}$. If $v^{\prime}=0$ then $v=v^{\prime \prime}$ $\in C_{a}\left(A, L_{2}+a\right)$. Then, suppose $v^{\prime} \neq 0$. Since $v^{\prime} \in C_{a_{1}}(W)$, there exist sequences $\left\{x_{v}^{\prime}\right\}$ of points of $W$ with $x_{v}^{\prime} \rightarrow a_{1}$ and $\left\{\lambda_{v}\right\}$ of complex numbers such that $\lambda_{v}\left(x_{v}^{\prime}-a_{1}\right) \rightarrow v^{\prime}$. Since $A \cap\left(L_{2}+a\right)=\{a\}$, there exists a sequence $\left\{x_{v}^{\prime \prime}\right\}$ of points of $L_{2}$ such that $x_{v}=x_{v}^{\prime}+x_{v}^{\prime \prime} \in A$ and $x_{v}^{\prime \prime} \rightarrow a_{2}=a-a_{1}$.

Setting

$$
y_{v}=v^{\prime \prime} / \lambda_{v}+x_{v}^{\prime \prime}, \quad v=1,2, \ldots,
$$

we see that $y_{v} \in L_{2}$ and $\lambda_{v}\left(y_{v}-x_{v}\right) \rightarrow v$, and so $v \in C_{0}\left(A, L_{2}\right)$. Therefore

$$
C_{a_{1}}(W)+L_{2} \subset C_{a}\left(A, L_{2}+a\right)
$$

which together with (2.8) gives (2.7).

The following four properties are obvious.

Property 2.5. $C_{a}(A, B)=C_{a}(B, A)=C_{0}(A-a, B-a)$.

Property 2.6. $C_{a}\left(A_{1} \cup A_{2}, B\right)=C_{a}\left(A_{1}, B\right) \cup C_{a}\left(A_{2}, B\right)$, provided $C_{a}\left(A_{1}, B\right)$ and $C_{a}\left(A_{2}, B\right)$ are well defined.

Property 2.7. If $B=\{a\}$ then $C_{a}(A, B)=C_{a}(A)$ is the taingent cone of $A$ at $a$. 
Property 2.8. If $A_{1} \subset A, B_{1} \subset B$, then $C_{a}\left(A_{1}, B_{1}\right) \subset C_{u}(A, B)$.

Since every analytic cone is algebraic, Lemma 2.1 gives

Property 2.9. If $A$ has the germ at a of pure dimension $p$ and $B$ has the germ at $a$ of pure dimension $q$, then $C_{a}(A, B)$ is an algebraic cone in $C^{m}$ of pure dimension $p+q$.

Property 2.10. $C_{a}(A)+C_{a}(B) \subset C_{a}(A, B)$.

If $C_{a}(A) \cap C_{a}(B)=\{0\}$ then $C_{a}(A)+C_{a}(B)=C_{a}(A, B)$.

This property may be proved by considering the mappings $f, \pi$ from the proof of Lemma 2.1 and by applying Lemmas $2.1,2.2,2.3$.

If $M$ is a complex manifold and $\varphi: U \rightarrow C^{m}$ is a map near $a \in M$, then the relative tangent cone $C_{a}(A, B)$ is defined to be the cone in the tangent space $T_{a} M$ defined by

$$
C_{a}(A, B)=\left(\varphi^{\prime}(a)\right)^{-1} C_{\varphi(a)}(\varphi(A), \varphi(B)) .
$$

It follows from Lemma 2.3 that this definition is independent of the map $\varphi$ chosen near $a$.

3. Proper intersection. We present here some facts needed in the sequel, concerning proper intersections at an isolated point. We suppose that paper [4] by Draper is known for the reader.

Now we recall the definition of the topology of local uniform convergence (for some more details, see e.g. [17]).

Let $X$ be a metric space and let $\mathscr{F}_{X}$ be the family of all closed subsets of $X$. The topology $\mathscr{T}_{X}$ on $\mathscr{F}_{X}$ generated by the sets

$$
\mathscr{U}(S, K)=\left\{F \in \mathscr{F}_{X} \mid F \cap K=\varnothing, F \cap U \neq \varnothing \text { for } U \in S\right\}
$$

corresponding to all compact subsets $K \subset X$ and all finite families $S$ of open subsets of $X$ is called the topology of local uniform convergence.

Let $M$ be a complex vector space of dimension $m$ and let $X, Y$ be locally analytic subsets of $M$ of pure dimensions $k$ and $m-k$, respectively. If \# ( $X \cap Y)$ $<\infty$ then we define

$$
{ }^{\#}(X \cdot Y)=\sum_{a \in X \cap Y} i(X \cdot Y ; a),
$$

where $i(X \cdot Y ; a)$ is the intersection multiplicity of $X$ and $Y$ at $a$, in the sense given in Draper's paper [4].

Remark. If $X, Y$ are analytic subsets of an open set $\Omega \subset M$ then

$$
{ }^{\#}(X \cdot Y)=\operatorname{deg}(X \cdot Y),
$$

where $\operatorname{deg}(X \cdot Y)$ is the degree of the 0 -cycle $X \cdot Y$ in $\Omega$. 
THEOREM 3.1 ([21], Theorem 8.2). Let $X$ be a bounded purely $k$-dimensional locally analytic subset of $M, Y$ a purely $(m-k)$-dimensional analytic subset of an open set $\Omega \subset M$ and let $T$ be the set of all biholomorphic mappings $f: \Omega \rightarrow f(\Omega) \subset M$ such that

$$
\bar{X} \subset f(\Omega) \quad \text { and } \quad f(Y) \cap \partial X=\varnothing
$$

with the topology of local uniform convergence, where $\partial X=\bar{X} \backslash X$. Then the mapping

$$
T \ni f \rightarrow^{\#}(X \cdot f(Y)) \in \boldsymbol{Z}
$$

is continuous.

THEOREM 3.2. Let $X$ be a bounded locally analytic subset of $M$ of pure dimension $k, \Omega$ an open subset of $M$ such that $\bar{X} \subset \Omega$ and let $Y_{v}, v=0,1, \ldots$, be a sequence of purely $(m-k)$-dimensional analytic subsets of $\Omega$.

If $Y_{v} \rightarrow Y_{0}$ in the topology of local uniform convergence in $\Omega$ and $Y_{0} \cap \partial X$ $=\varnothing$, then there exists $v_{0} \in \boldsymbol{Z}_{+}$such that

$$
{ }^{\#}\left(Y_{v} \cdot X\right) \geqslant{ }^{*}\left(Y_{0} \cdot X\right) \quad \text { for } v \geqslant v_{0} .
$$

Proof. Since $\partial X$ is compact, $Y_{v} \rightarrow Y_{0}$ and $Y_{0} \cap \partial X=\emptyset$, there exist $r>0$ and $\gamma \in Z_{+}$such that

(i) $\Omega+z \supset \bar{X}$ and

(ii) $\left(Y_{v}+z\right) \cap \partial X=\varnothing$, provided $z \in B(r)$ and $v \geqslant \gamma$ or $v=0$.

Thus, by Theorem 1.1,

$$
{ }^{\#}\left(\left(Y_{v}+z\right) \cdot X={ }^{*}\left(Y_{v} \cdot X\right) \quad \text { for } z \in B(r), v=0 \text { or } v \geqslant \gamma .\right.
$$

Then (see e.g. [21], Lemma 6.1) we can choose $z_{0} \in B(r)$ such that $Y_{0}+z_{0}$ meet only transversally $X$. Therefore,

$$
\#\left(\left(Y_{0}+z_{0}\right) \cap X\right)={ }^{\#}\left(Y_{0} \cdot X\right) .
$$

Since $\left(Y_{y+\nu}+z_{0}\right) \rightarrow\left(Y_{0}+z_{0}\right)$ in $\tilde{\Omega}=\left(\Omega+z_{0}\right) \backslash \partial X$ as $v \rightarrow \infty$ by the continuity of intersection ([17], Theorem 3), we have

$$
\#\left(X \cap\left(Y_{v}+z_{0}\right)\right) \geqslant \#\left(X \cap\left(Y_{0}+z_{0}\right)\right) \text { for big enough } v,
$$

and so we have (3.2).

4. Intersection of locally analytic sets with submanifolds. Let $X$ be a purely $k$-dimensional analytic subset of a complex manifold $M$ of dimension $m$. Let $N$ be a submanifold of $M$ of dimension $n$ such that $N$ intersects $X$ at an isolated point $a \in M$. We shall denote by $\mathscr{F}_{a}(X, N)$ the set of all locally analytic subsets $V$ of $M$ satisfying the following three conditions: 
(i) $V$ has pure dimension $m-k$,

(ii) $N_{a} \subset V_{a}$, where $N_{a}, V_{a}$ denote the germs at $a$ of $N$ and $V$, respectively, (iii) $a$ is an isolated point of $V \cap X$.

The set $\mathscr{F}_{a}(X, N)$ is not empty and the intersection of $X$ and $V$ is proper at $a$. We define

$$
\begin{aligned}
\tilde{i}(X \cdot N ; a) & =\min \left\{i(X \cdot V ; a) \mid V \in \mathscr{F}_{a}(X, N)\right\}, \\
\mathscr{P}_{a}(X, N) & =\left\{V \in \mathscr{\mathscr { F }}_{a}(X, N) \mid i(X \cdot V ; a)=\tilde{i}(X \cdot N ; a)\right\} .
\end{aligned}
$$

The main purpose of this part is to characterize the set $\mathscr{P}_{a}(X, N)$.

Remark 4.1. The definitions of $\tilde{i}(X \cdot N ; a)$ and $\mathscr{P}_{a}(X, N)$ are local and they are invariant with respect to biholomorphic transformations. More precisely, we have:

(1) $\tilde{i}(X \cdot N ; a)$ depends only on the germs $X_{a}, N_{a}$,

(2) belonging of $V$ to $\mathscr{F}_{a}(X, N)$ or $\mathscr{P}_{a}(X, N)$ depends only on the germ $V_{a}$,

(3) if $M^{\prime}$ is a manifold of dimension $m, F: M \rightarrow M^{\prime}$ is a biholomorphic mapping, $X^{\prime}=F(X), N^{\prime}=F(N), V^{\prime}=F(V)$ and $a^{\prime}=F(a)$, then

(a) $\tilde{i}(X \cdot N ; a)=\tilde{i}\left(X^{\prime} \cdot N^{\prime}, a^{\prime}\right)$,

(b) $V \in \mathscr{F}_{a}(X, N)$ if and only if $V^{\prime} \in \mathscr{F}_{a^{\prime}}\left(X^{\prime}, N^{\prime}\right)$ and

(c) $V \in \mathscr{P}_{a}(X, N)$ if and only if $V^{\prime} \in \mathscr{P}_{a^{\prime}}\left(X^{\prime}, N^{\prime}\right)$.

This remark gives possibility to transport our consideration to a complex vector space and $a=0$. Then, let $M$ be a complex vector space of dimension $m$ and let $N, L$ be linear subspaces of $M$ of dimension $n$ and $m-n$, respectively, such that $M=N+L$. Since the sum is direct, the projection

$$
\pi: M=L+N \ni x+y \rightarrow x \in L
$$

is well defined.

Let $D$ and $G$ be open balls centered at 0 in $L$ and $N$, respectively. Suppose that $X, V$ are analytic subsets of $\Omega=D+G$ of pure dimension $k$ and $m-k$, respectively, such that $G \subset V, V \cap X=\{0\}, \pi \mid X: X \rightarrow D$ is proper and the set $Y=V \cap D$ has the pure dimension $m-n-k$.

Proposition 4.2. If $V \in \mathscr{P}_{0}(X, N)$ then

(i) $Y+G \in \mathscr{P}_{0}(X, N)$,

(ii) the germ $Y_{0}$ of $Y$ at 0 is the germ of a submanifold of $M$,

(iii) $C_{0}(Y) \cap C_{0}(\pi(X))=\{0\}$ and

(iv) $\tilde{i}(X \cdot N ; 0)=\operatorname{deg}_{0} \pi[X]$.

Proof. To prove (i) we set

$d=\tilde{i}(X \cdot N ; 0)=i(V \cdot X ; 0) \quad$ and $\quad X_{\varepsilon}=X \cap((\varepsilon D)+G)$ for $\varepsilon \in(0,1)$. 
Note that $\bar{X}_{\varepsilon} \subset \Omega$ and $\partial X_{\varepsilon} \cap V=\varnothing$. By compactness of $\bar{X}_{\varepsilon}$ and $\partial X_{\varepsilon}$ there exists, for a fixed $\varepsilon \in(0,1), \delta \in(0,1)$ such that

$$
\Omega+v \supset \bar{X}_{\varepsilon} \quad \text { and } \quad(V+v) \cap \partial X_{\varepsilon}=\varnothing \quad \text { for } v \in \delta G .
$$

Then, by Theorem 3.1,

$$
{ }^{\#}\left((V+v) \cdot X_{\varepsilon}\right)=d \quad \text { for } v \in \delta G .
$$

Moreover, $(V+v) \in \mathscr{F}_{0}(X, Y)$ for $v \in \delta G$. Since also $V \in \mathscr{P}_{0}(X, N), i\left((V+v) \cdot X_{c}, 0\right)$ $=d$. Thus we have

$$
(V+v) \cap \bar{X}_{\varepsilon}=\{0\} \quad \text { for } v \in \delta G,
$$

and so we can pick $\varepsilon>0$ small enough such that $\bar{X}_{\varepsilon} \subset \varepsilon D+\delta G$.

For any $t \geqslant 1$, the set

$$
V_{t}=\{x+t y \in D+t G \mid x+y \in V\}
$$

is an analytic subset of $D+t G, D+t G \supset \bar{X}_{\varepsilon}$ and

$$
\bar{X}_{\varepsilon} \cap V_{t}=\{0\} \quad \text { for } t \geqslant 1 \text {. }
$$

Indeed, if $x+t y \in \bar{X}_{\varepsilon}$ and $x+y \in V$ then $t y \in \delta G$. Hence, $v=(t y-y)=$ $(t-1) y \in \delta G$, and so $x+t y \in \bar{X}_{\varepsilon} \cap(V+v)$. Thus, by (4.1), we have $x+t y=0$. Then, by Theorem 3.1,

$$
i\left(X_{\varepsilon} \cdot V_{t} ; 0\right)=d \quad \text { for } t \geqslant 1 .
$$

Letting

$$
V^{v}=V_{v} \cap \Omega \quad \text { for } v=1,2, \ldots
$$

we obtain that

$$
i\left(X_{\varepsilon} \cdot V^{v}, 0\right)={ }^{\#}\left(X_{\varepsilon} \cdot V^{v}\right)=d .
$$

Since $V^{v} \rightarrow V^{0}=Y+G$ (in the topology of local uniform convergence) and $V^{0} \cap \bar{X}_{\varepsilon}=\{0\}$, by Theorem 3.2 we have

$$
i\left(V^{0} \cdot X_{\varepsilon}, 0\right)={ }^{\#}\left(V^{0} \cdot X_{\varepsilon}\right) \leqslant{ }^{\#}\left(V_{v} \cdot X_{\varepsilon}\right)=d .
$$

Thus $i\left(V^{0} \cdot X_{\varepsilon} ; 0\right) \leqslant d$, and so $V_{0}=Y+G \in \mathscr{P}_{0}(X, N)$. This ends the proof of (i).

Let $Z$ be an analytic subset of the ball $D$ of pure dimension $m-n-k$ such that 0 is an isolated point of $Z \cap \pi(X)$ and let $\widetilde{V}=Z+G$. Then, using Theorem 5.3. from [4],

$$
i(X \cdot \tilde{V} ; 0)=i(\pi[X] \cdot Z ; 0) \geqslant \operatorname{deg}_{0} \pi[X]
$$

with equality if and only if $Z_{0}$ is the germ of a submanifold such that $T_{0} Z \cap C_{0}(\pi(X))=\{0\}$. Since $\tilde{V} \in \mathscr{F}_{0}(X, N)$ and $Y+G \in \mathscr{P}_{0}(X, N)$, next points of Proposition 4.2 are now clear.

Lemma 4.3. Let $M$ be a complex vector space of dimension $m, N$ a linear 
subspace of $M$ of dimension $n<m$ and let $V_{0}$ be a purely $(m-k)$-dimensional germ at 0 of a locally analytic subset $V$ of $M$ such that $N_{0} \subset V_{0}$. If $m-n-k>0$, then there exists a linear subspace $L$ of $M$ of dimension $m-n$ such that

(a) $L+N=M$,

(b) $\operatorname{dim}_{0}(L \cap V)=m-n-k$ and

(c) $\operatorname{deg}_{0}(L \cap V)=\operatorname{deg}_{0} V$.

Proof. Let $\eta, \xi$ be linear subspaces of $M$ of dimensions $k, m-k$, respectively, such that $\xi \supset N, \eta \cap C_{0}(V)=\{0\}$ and $\xi+\eta=M$.

If $\pi$ is the projection onto $\xi$ parallel to $\eta$, then $\pi \mid V: V \rightarrow \xi$ is proper in a small enough neighbourhood of the point 0 . Then, there exists a linear subspace $\zeta$ of $\xi$ of dimension $m-n-k$ such that $\zeta \cap N=\{0\}$ and $\zeta$ contains at least one unramified point of the branched covering $\pi \mid V$. Thus, for $L=\zeta+\eta$, (a), (b), (c) are satisfied.

Now we turn to the characterization of the family $\mathscr{P}_{a}(X, N)$.

THEOREM 4.4. If $V \in \mathscr{F}_{a}(X, N)$ then $V \in \mathscr{P}_{a}(X, N)$ if and only if $V_{a}$ is the germ of a submanifold and

$$
C_{a}(X, N) \cap T_{a} V=T_{a} N .
$$

Proof. If $k+n=m$ then Theorem 4.4 is trivial. If $n=0$ then the theorem is well known (see e.g. [4], Theorem 6.3). Then, we shall consider now the case $k+n<m, n>0, k>0$.

To prove the necessity let us fix $V \in \mathscr{P}_{a}(X, N)$. Without loss of generality we may assume that $M$ is a complex normed vector space of dimension $m$, $N$ a linear subspace of $M$ of dimension $n$ and that $a=0$. We pick now a linear subspace $L$ of $M$ of dimension $m-n$ such that conditions (a), (b), (c) of Lemma 4.3 are satisfied. By Proposition 4.2, the germ $(L \cap V)_{0}$ is the germ of a submanifold, and so $\operatorname{deg}_{0} V=1$. Therefore, $V$ is the germ at 0 of a submanifold of $M$ and, also by Proposition 4.2, $T_{0}(L \cap V) \cap C_{0}(\pi(X))=\{0\}$ where $\pi: L+N \supset x+y \rightarrow x \in L$ is the natural projection.

Since $T_{0} V=T_{0}(L \cap V)+N$ and $C_{0}(X, N)=C_{0}(\pi(X j)+N$,

$$
T_{0} V \cap C_{0}(X, N)=N=T_{0} N,
$$

and so the necessity is proved.

To prove the sufficient condition, we can assume that $M, N$ satisfy the assumptions of Proposition 4.2 and $V$ is a linear subspace of $M$ of dimension $m-k$. We can now choose, for a fixed element of $\mathscr{P}_{a}(X, N)$, a linear subspace $L$ of $M$ satisfying conditions (a), (b), (c) of Lemma 4.3. Then, by Proposition 4.2, we have

$$
\tilde{i}(X \cdot N ; 0)=\operatorname{deg}_{0} \pi[X]
$$

with the same $\pi$ as in Proposition 4.2.

Since $V=L \cap V+N$ and (b),

$$
T_{0}(L \cap V) \cap C_{0}(\pi(X))=\{0\} .
$$


Thus ([4], Theorem 5.3),

$$
i(V \cdot X ; 0)=i(\pi[X] \cdot(L \cap V), 0)=\operatorname{deg}_{0} \pi[X]
$$

and by (4.2) we have

$$
i(V \cdot X ; 0)=\tilde{i}(X \cdot N ; 0),
$$

and so $V \in \mathscr{P}_{0}(X, N)$ ending the proof.

Let as now assume $M, N, L, D, G, \Omega$, and $\pi$ to be the same as in Proposition 4.2 and let $X$ be an analytic subset of $\Omega$ of pure dimension $k$ such that $\pi \mid X: X \rightarrow D$ is proper and $X \cap G=\{0\}$.

Remark 4.5. $\tilde{i}(X \cdot N ; 0)=\operatorname{deg}_{0} \pi[X]$.

Remark 4.6. If $X=X_{1} \cup \ldots \cup X_{k}$ is the decomposition of $X$ into irreducible components, then

$$
\tilde{i}(X \cdot N ; 0)=\sum_{j=1}^{k} \tilde{i}\left(X_{j} \cdot N ; a\right) .
$$

In the natural way we extend the definition of $\tilde{i}$ to the case $a \notin X \cap N$ by taking

$$
\tilde{i}(X \cdot N ; a)=0 \quad \text { whenever } a \notin X \cap N .
$$

Then, formula (4.3) makes sense even when a component $X_{j}$ does not pass through 0 .

Remark 4.7. If $N=\{a\}$ then $\tilde{i}(X \cdot N ; a)=\operatorname{deg}_{a} X$ and Theorem 4.4 can be seen as a generalization of Theorem 6.3 in [4].

5. Multiplicity of isolated intersection. Let $X, Y$ be locally analytic subsets of a complex manifold $M$ such that $a$ is an isolated point of $X \cap Y$. We define the diagonal mapping $\delta: M \ni x \rightarrow(x, x) \in M \times M$ and the diagonal $\Delta_{M}=\delta(M)$ of $M$.

Definition 5.1. The positive integer

$$
i(X \cdot Y ; a)=\tilde{i}\left((X \times Y) \cdot \Delta_{M} ; \delta(a)\right)
$$

is defined to be the multiplicity of intersection of $X$ and $Y$ at $a$.

THEOREM 5.2. We have

(1) $i(X \cdot Y ; a)=i(Y \cdot X ; a)$,

(2) $i(X \cdot Y ; a)$ depends only on germs $X_{a}, Y_{a}$.

(3) If $\varphi: M \rightarrow \tilde{M}$ is a biholomorphic mapping, then $i(X \cdot Y ; a)=i(\varphi(X) \cdot \varphi(Y)$; $\varphi(a))$ and

(4) If $X_{1}, \ldots, X_{s}$ are all components of $X$ passing through $a$, then

$$
i(X \cdot Y ; a)=\sum_{j=1}^{s} i\left(X_{j} \cdot Y, a\right) .
$$


Proof. Ad (1). Since $\psi: M \times M \ni(x, y) \rightarrow(y, x) \in M \times M$ is a biholomorphic mapping,

$$
\begin{aligned}
i(X \cdot Y ; a) & =\tilde{i}\left((X \times Y) \cdot \Delta_{M} ; \delta(a)\right)=\tilde{i}\left(\psi(X \times Y) \cdot \psi\left(\Delta_{M}\right) ; \delta(a)\right) \\
& =\tilde{i}\left((Y \times X) \cdot \Delta_{M} ; \delta(a)\right)=i(X \cdot Y ; a) .
\end{aligned}
$$

Ad(2). This is trivial.

Ad(3). Since

$$
\varphi \times \varphi: M \times M \ni(x, y) \rightarrow(\varphi(x), \varphi(y)) \in \tilde{M} \times \tilde{M}
$$

is a biholomorphic mapping,

$$
\begin{aligned}
i(X \cdot Y ; a) & =\tilde{i}\left((X \times Y) \cdot \Delta_{M} ; \delta(a)\right) \\
& =\tilde{i}\left(((\varphi \times \varphi)(X \times Y)) \cdot\left((\varphi \times \varphi)\left(\Delta_{M}\right)\right) ; \delta(\varphi(a))\right) \\
& =\tilde{i}\left((\varphi(X) \times \varphi(Y)) \cdot \Delta_{\tilde{M}} ; \delta(\varphi(a))\right) \\
& =i(\varphi(X) \cdot \varphi(Y) ; \varphi(a)) .
\end{aligned}
$$

Ad(4). We have

$$
i(X \cdot Y ; a)=\tilde{i}\left(\left(X \times Y \cdot \Delta_{M} ; \delta(a)\right)=\sum_{j=1}^{s} \tilde{i}\left(\left(X_{j} \times Y\right) \cdot \Delta_{M} ; \delta(a)=\sum_{j=1}^{S} i\left(X_{j} \cdot Y ; a\right) .\right.\right.
$$

In the case where $Y$ is a submanifold the definition of $i(X \cdot Y ; a)$ presented above overlaps with $\tilde{i}(X \cdot Y ; a)$ which was introduced earlier.

Proposition 5.3. If a locally analytic subset $X$ of a manifold $M$ intersects a submanifold $N$ of $M$ at an isolated point $a$, then

$$
i(X \cdot N ; a)=\tilde{i}(X \cdot N ; a) .
$$

Proof. By Theorem 5.2, (4), we may assume that $X$ and the germ $X_{a}$ of $X$ at $a$ are irreducible. Moreover, we may assume that:

(a) $M$. is a complex vector space of dimension $m$,

(b) $N$ is a subspace of $M$ of dimension $n$,

(c) $a=0$,

(d) $L$ is a $(m-n)$-dimensional subspace of $M$ such that $L+N=M$,

(e) $D$ is an open ball in $L$ centered at 0 ,

(f) $X$ is a $k$-dimensional irreducible and locally irreducible analytic subset of $D+N$ such that $N \cap X=\{0\}$ and

$$
\pi \mid X: X \ni x+y \rightarrow x \in D
$$

is proper.

We have

$$
i(X \cdot N ; 0)=\tilde{i}\left((X \times N) \cdot \Delta_{M} ; 0\right) .
$$


The mapping

$$
F: M \times M \ni(x, y) \rightarrow(x-y,-y) \in M \times M
$$

is a linear isomorphism an $F(X \times N)=(X \times\{0\})+\Delta_{N}, \quad F\left(\Delta_{M}\right)=\{0\} \times M$, $F(0)=0$. Thus

$$
i(X \cdot N ; 0)=\tilde{i}(Y \cdot(\{0\} \times M) ; 0),
$$

where $Y=(X \times\{0\})+\Delta_{N}$.

The restriction $p \mid Y: Y \rightarrow D+N$ of the natural projection $p: M \times M$ $\ni(x, y) \rightarrow x \in M$ is a proper mapping. Since $Y \cap(\{0\} \times M)=\{0\}$, by Remark 4.5,

$$
\tilde{i}(Y \cdot(\{0\} \times M) ; 0)=\operatorname{deg}_{0} p[Y] .
$$

Since also $\operatorname{deg}_{0} p[Y]=\operatorname{deg}_{0} \pi[X]$, we have

$$
i(X \cdot N ; 0)=\operatorname{deg}_{0} \pi[X]
$$

and so, by Remark 4.5 ,

$$
i(X \cdot N ; 0)=\tilde{i}(X \cdot N ; 0)
$$

ends the proof.

Let $N$ be a submanifold of a submanifold $M$ and let $X, Y$ be two locally analytic subsets of $N$. If $a$ is an isolated point of $X \cap Y$ we can calculate $i(X \cdot Y ; a)$ regarding $X, Y$ as subsets of $N$ or subsets of $M$. Thus, we may obtain two numbers $i_{N}(X \cdot Y ; a)$ and $i_{M}(X \cdot Y ; a)$.

Proposition 5.4. We have

$$
i_{N}(X \cdot Y ; a)=i_{M}(X \cdot Y ; a) .
$$

Proof. Without loss of generality we may assume that $M, N$, a satisfy conditions (a), (b), (c) presented in the proof of Proposition 5.3 with $n<m$, and that $X, Y$ are irreducible and locally irreducible with $X \cap Y=\{0\}$.

We see that

$$
M \times M=(M \times\{0\})+\Delta_{M}, \quad N \times N=(N \times\{0\})+\Delta_{N}
$$

and

$$
\begin{gathered}
\pi_{M}: M \times M \ni(x, y) \rightarrow(x-y, 0) \in M \times\{0\}, \\
\pi_{N}: N \times N \ni(x, y) \rightarrow(x-y, 0) \in N \times\{0\}
\end{gathered}
$$

are projections parallel to $\Delta_{M}, \Delta_{N}$, respectively. Fixing a norm in $M \times M$, we can find an open neighbourhood $Z$ of 0 in $X \times Y$ and balls $B_{M}(r)$ and $B_{N}(r)$ in $M \times\{0\}$ and $N \times\{0\}$, respectively, such that the mappings

$$
\pi_{M}\left|Z: Z \rightarrow B_{M}(r), \quad \pi_{N}\right| Z: Z \rightarrow B_{N}(r)
$$

are proper. By Remark 4.5, 


$$
i_{N}(X \cdot Y ; 0)=\operatorname{deg}_{0} \pi_{N}[Z] \quad \text { and } \quad i_{M}(X \cdot Y, 0)=\operatorname{deg}_{0} \pi_{M}[Z] \text {. }
$$

Since $\pi_{N}[Z]$ and $\pi_{M}[Z]$ are the same cycles considered only in two different ambient manifolds,

$$
\operatorname{deg}_{0} \pi_{N}[Z]=\operatorname{deg}_{0} \pi_{M}[Z] .
$$

Let $X$ be a locally analytic subset of a manifold $M$ and let $a \in X$. If $X_{1}, \ldots, X_{s}$ are all components of $X$ passing through $a$, then the numbers $\operatorname{deg}_{a} X_{1}, \ldots, \operatorname{deg}_{a} X_{s}$ are well defined. We extend the definition of the degree from the pure dimensional case, which is known in the literature, to the general case.

Definition 5.5. The number $\operatorname{deg}_{a} X$ defined by

$$
\operatorname{deg}_{a} X=\operatorname{deg}_{a} X_{1}+\ldots+\operatorname{deg}_{a} X_{s}
$$

is called the degree of $X$ at $a$.

THEOREM 5.6. If $X, Y$ are locally analytic subsets of $a$ manifold $M$ and $a$ is an isolated point of $X \cap Y$, then

$$
i(X \cdot Y ; a) \geqslant \operatorname{deg}_{a} X \cdot \operatorname{deg}_{a} Y
$$

with equality if and only if

$$
C_{a}(X) \cap C_{a}(Y)=\{0\} .
$$

Proof. It is clear that in order to prove Theorem 5.6 it suffices to prove it under assumptions that $X$ and $Y$ are irreducible.

By definition we have

$$
i(X \cdot Y ; a)=\tilde{i}\left((X \times Y) \cdot \Delta_{M} ; \delta(a)\right) .
$$

Let $V \in \mathscr{P}_{\delta(a)}\left(X \times Y, \Delta_{M}\right)$. Of course,

$$
i(X \cdot Y ; a)=i((X \times Y) \cdot V ; \delta(a)) \geqslant \operatorname{deg}_{\delta(a)}(X \times Y)=\operatorname{deg}_{a} X \cdot \operatorname{deg}_{a} Y
$$

with equality if and only if

$$
C_{\delta(a)}(X \times Y) \cap T_{\delta(a)} V=\{0\} .
$$

Then, we see that

(a) if (5.1) holds then $C_{\delta(a)}(X \times Y) \cap T_{\delta(a)} \Delta_{M}=\{0\}$, and so $C_{a}(X) \cap$ $C_{a}(Y)=\{0\}$

(b) if $C_{a}(X) \cap C_{a}(Y)=0$ then, by Property 2.10 ,

$$
C_{\delta(a)}\left(X \times Y, \Delta_{M}\right)=C_{\delta(a)}(X \times Y)+T_{\delta(a)} \Lambda_{M}
$$

and

$$
C_{\delta(a)}(X \times Y) \cap T_{\delta(a)} \Delta_{M}=\{0\}
$$


Since $V \in \mathscr{P}_{\delta(a)}\left(X \times Y, \Delta_{M}\right)$, by Theorem 4.4,

$$
C_{\delta(a)}\left(X \times Y, \Delta_{M}\right) \cap T_{\delta(a)} V=T_{\delta(a)} \Delta_{M},
$$

and so we have (5.1) which ends the proof.

6. Intersection multiplicity via local algebra (after $\mathrm{P}$. Samuel). Let $(A, \mathrm{~m})$ be a $d$-dimensional Noetherian local ring, $\mathscr{M}$ a finitely generated $A$-module and $q$ an m-primary ideal. Then it is well known (see e.g. [23], Vol. II) that the Hilbert-Samuel function length ${ }_{A}\left(\mathscr{M} / q^{n+1} \mathscr{M}\right)$ can be expressed, for all large $n$, as a polynomial in $n$ with rational coefficients, and degree equal to $\operatorname{dim} \mathscr{M}$, and therefore at most $d$. Since this polynomial can only take integer values for all large $n$, it can be written in the form

$$
\frac{e}{d !} n^{d}+\text { terms of lower degree }
$$

with a nonnegative integer $e=e(q, \mathscr{M})$. We have

$$
e(q, \mathscr{M})=\lim _{n \rightarrow \infty} \frac{d !}{n^{d}} \text { length }_{A}\left(\mathscr{H} / q^{n+1} \mathscr{M}\right) .
$$

The notation being as in Section 4 , we denote by $\left(\mathscr{G}_{M, a}, \mathrm{~m}_{a}\right)$ and $\left(\mathcal{O}_{X, a}, \mathrm{~m}_{X, a}\right)$ the local rings of germs at the point $a$ of holomorphic functions defined in a neighbourhood of $a$ in $M$ and $X$, respectively. Let $I_{N, a}$ be the ideal in $\mathcal{O}_{M, a}$ defining $N$ at $a$.

PROPOSITION 6.1. Let $\mathscr{F}$ be the set of all ideals $J$ in $\mathcal{O}_{M . a}$ satisfying

(i) $J \subset I_{N, a}$ and

(ii) $J \cdot \mathscr{O}_{X, a}$ is $\mathrm{m}_{X, a}$-primary,

and put

$$
\begin{aligned}
\mu_{1} & =\min \left\{e\left(J \cdot \mathcal{O}_{X, a}\right) \mid J \in \mathscr{F}\right\}, \\
\mu_{2} & =\min \left\{e\left(J \cdot \mathscr{O}_{X . a}\right) \mid J \in \mathscr{F} \text { and } \operatorname{dim}\left(\mathcal{O}_{M . a} / J\right)=m-k\right\}, \\
\mu_{3} & =\min \left\{e\left(J \cdot \mathcal{O}_{X, a}\right) \mid J \in \mathscr{F}, \operatorname{dim}\left(\mathcal{O}_{X, a} / J\right)=m-k\right. \\
& \text { and } J \text { is generated by } k \text { elements }\} .
\end{aligned}
$$

Then

$$
\tilde{i}(X \cdot N ; a)=\mu_{1}=\mu_{2}=\mu_{3}=e\left(I_{N, a} \cdot \mathscr{C}_{X . a}\right) .
$$

Proof. If $J \in \mathscr{F}, \operatorname{dim}\left(\mathcal{O}_{M, a} / J\right)=m-k$, and $J$ is generated by $k$ elements, then the germ $V$, which is defined by $J$, is the complete intersection which intersects the germ of $X$ at $a$ properly. Therefore, $i(X \cdot V ; a)=e\left(J \mathcal{U}_{X, a}\right)$ by the coincidence of the different multiplicities of intersection in the classical case of a proper intersection (see e.g. [14]) and by Samuel's reduction theorem. This shows that 
Obviously,

$$
\mu_{1} \leqslant \tilde{i}(X \cdot N ; a) \leqslant \mu_{3} .
$$

$$
\mu_{1} \leqslant \mu_{2} \leqslant \mu_{3} \quad \text { and } \quad e\left(I_{N, a} \cdot \mathcal{O}_{X, a}\right) \leqslant \mu_{1} .
$$

It remains to prove

$$
\mu_{3} \leqslant e\left(I_{N, a} \cdot \mathcal{C}_{X, a}\right) .
$$

But this follows from the fact that there exists an $m_{X, a}$-primary ideal $q \subset I_{N, a} \cdot \mathcal{O}_{X, a}$ generated by a system of parameters $x_{1}, \ldots, x_{k}$ of $\mathcal{O}_{X, a}$ such that

$$
e\left(I_{N, a} \cdot \mathcal{O}_{X, a}\right)=e\left(\left(x_{1}, \ldots, x_{k}\right) \cdot \mathcal{O}_{X, a}\right)
$$

(see e.g. [23], Vol. II, p. 294).

Remark 6.2. The proof of the theorem mentioned in [23] shows even more. It shows that for every superficial sequence $l_{1}, \ldots, l_{k}$ of order 1 for $I_{N, a} \cdot \mathcal{O}_{X, a}$ one has

$$
e\left(\left(l_{1}, \ldots, l_{k}\right) \mathcal{O}_{X, a}\right)=e\left(I_{N, a} \cdot \mathcal{O}_{X, a}\right)=\tilde{i}(X \cdot N, a) .
$$

Then, we can see Theorem 4.4 as a geometric necessary condition for $l_{1}, \ldots, l_{k}$ to be a superficial sequence of order 1 for $I_{N, a} \cdot \mathcal{O}_{X, a}$.

Now, suppose that, as in Section 5, two locally analytic subsets $X$ and $Y$ of a complex manifold $M$ intersect properly in an isolated point $a \in X \cap Y$. Let $I_{\Delta, \delta(a)}$ be the ideal of the germ of the diagonal $\Delta_{M}$ at $(a, a)=\delta(a)$ in the ring $\mathcal{O}_{M, a} \hat{\otimes}_{c} \mathcal{O}_{M, a}$, where $\hat{\otimes}_{c}$ means the analytic tensor product (see e.g. [9]).

By Proposition 6.1 and by the definition of $i$ via $\tilde{i}$ and since $\mathscr{C}_{X \times Y, \delta(a)} \cong$ $\mathcal{O}_{X, a} \otimes_{c} \mathcal{O}_{Y, a}$, we have

Proposition 6.3.

$$
i(X \cdot Y, a)=e\left(I_{\Delta, \delta(a)} \cdot\left(\mathcal{O}_{X, u} \hat{\otimes}_{c} \mathcal{O}_{Y, a}\right)\right)
$$

By Propositions 6.1, 6.2 and 5.3 we have

Proposition 6.4. If a locally analytic subset $X$ of a manifold $M$ intersects a submanifold $N$ of $M$ in an isolated point a, then

$$
e\left(I_{N, a} \cdot \mathcal{O}_{X, a}\right)=e\left(I_{\Delta, \delta(a)} \cdot\left(\mathcal{O}_{X, a} \hat{\otimes}_{C} \mathcal{O}_{N, a}\right)\right) .
$$

Of course, Proposition 6.4 may also be proved in a pure algebraic way.

Remark 6.5. The reduction theorem of [13] for proper intersection of $X$ and $Y$ at an isolatad point $a$ says that whenever $I_{Y, a} \subset \mathcal{O}_{M, a}$ is a complete intersection ideal, then the reduction to the diagonal is not necessary, that is,

$$
i(X \cdot Y ; a)=e\left(I_{Y, a} \cdot \mathcal{O}_{X, a}\right) .
$$

In our extension to improper intersections we assumed that $Y=N$ is a submanifold at $a$.

The following example shows that for an arbitrary "non-linear" complete intersection $Y$ at the point $a$, the extension of Samuel's reduction theorem to improper intersection may fail. 
Example 6.6. Let $M=C^{3}, X=\{0\}$ in $C^{3}$ and let $Y$ be the union of two lines $x_{1}=x_{2}=0$ and $x_{1}=x_{3}=0$. The origin $0=a$ is then an isolated point of the improper intersection $X \cap Y$ and we have $e\left(I_{Y, a} \cdot \mathcal{O}_{X, a}\right)=1$ but

$$
e\left(I_{\Delta, \delta(a)} \cdot \mathcal{O}_{X \times Y, \delta(a)}\right)=\tilde{i}\left((X \times Y) \cdot \Delta_{M} ; \delta(a)\right)=2 .
$$

\section{References}

[1] R. Achilles, On the intersection multiplicity of improper components in algebraic geometry, Beitr. Algebra Geom. 19 (1985), 113-129.

[2] E.-A. Behrens, Zur Schnittmultiplizität uneigentlicher Komponenten in der algebraischen Geometrie, Math. Z. 55 (1952), 119-215.

[3] S. Diaz, Space curves that intersect often, Pacific J. Math. 123.2 (1986), 263-267.

[4] R. Draper, Intersection theory in analytic geometry, Math. Ann. 180 (1969), 175-204.

[5] W. Fulton, Intersection theory, Ergebnisse der Math., 3. Folge, Springer-Verlag, 1984.

[6] L. J. van Gastel, Excess intersections in projective space, Banach Center Publications, Topics in Algebra, vol. 26, PWN-Polish Scientific Publishers, Warsaw 1990 (to appear).

[7] -, Private letter to T. Winiarski dated April 22. 1988.

[8] -, A geometric approach to Vogel's intersection theory, University of Utrecht, Preprint 427 (1986).

[9] H. Grauert and R. Remmert, Analytische Stellenalgebren, Grundlehren der Math. 176, Springer-Verlag, 1971.

[10] M. Nagat a, Note on intersection multiplicity of proper components of algebraic or algebroid varieties, Mem. Coll. Sci. Univ. Kyoto, Ser. A. Math. 28 (1954), 279281.

[11] -, Local rings, Intersciences, New York 1962.

[12] P. Sa muel, La notion de multiplicité en algèhre et en géométrie algébrique, J. Math. Pures Appl. 9.30 (1951), 159-274.

[13] -, Méthodes d'algèbre abstraite en géométrie algébrique, Ergebnisse der Mathematik, Neue Folge, Springer-Verlag, 1955.

[14] E. Selder, Eine algebraische Definition lokaler analytischer Schnittmultiplizitäten, Rev. Roumaine Math. Pures Appl. 5. 29 (1984), 417-432.

[15] F. Severi, Il concetto generale di molteplicita delle soluzioni pei sistemi equazioni algebriche e la teoria dell eliminazione, Ann. di Mat. (4) 26 (1947), 221.270.

[16] J. Stückrad and W. Vogel, An algebraic approach to the intersection theory, in: The curves seminar at Queen's, Vol. II, 1-32, Queen's papers in pure and applied mathematics, No. 61, Kingston, Ontario, Canada, 1982.

[17] P. Tworzewski and T. Winiarski, Continuity of intersection of analytic sets, Ann. Polon. Math. 42 (1983), 387-393.

[18] W. Vogel, Lectures on results on Bézout's Theorem, Notes by D. P. Patil, Lectures Notes, Tata Institute of Fundamental Research of Bombay, Springer-Verlag, 1984.

[19] A. Weil, Foundations of algebraic yeometry, 1946, Revised and enlarged edition, Amer. Math. Soc. Coll. Publ. 29 (1962).

[20] H. Whitney, Tangents to an analytic variety, Ann. of Math. 81 (1965), 496-549.

[21] T. Winiarski, Continuity of total number of intersection, Ann. Polon. Math. 47 (1986), 155-178.

[22] -, Local properties of intersection, Kluwer Acad. Comp., Deformations of Math. Structures (1989), $141-150$.

[23] O. Zarisk i and P. Sa muel, Commutative Algebra, Vol. I and II, D. van Nostrand Comp., Princeton 1958 and 1960. 